\title{
Vitamin D-binding protein contributes to COPD by activation of alveolar macrophages
}

\author{
A M Wood, ${ }^{1}$ C Bassford, ${ }^{1}$ D Webster, ${ }^{1}$ P Newby, ${ }^{1}$ P Rajesh, ${ }^{2}$ R A Stockley, ${ }^{3}$ \\ D R Thickett ${ }^{1}$
}

See Editorial, p 189

- Additional materials are published online only. To view these files please visit the journal online (http://thorax.bmj. com).

${ }^{1}$ School of Clinical and Experimental Medicine, University of Birmingham, UK ${ }^{2}$ Heart of England NHS Trust, Bordesley Green, Birmingham, UK

${ }^{3}$ Lung Investigation Unit, University Hospitals Birmingham, Birmingham, UK

\section{Correspondence to}

A M Wood, School of Clinical and Experimental Medicine, University of Birmingham, Birmingham B15 2TT, UK; a.m.wood@bham.ac.uk

Received 19 April 2010 Accepted 2 November 2010 Published Online First 12 January 2011

\begin{abstract}
Background Vitamin D-binding protein (DBP) genetic polymorphisms have been associated with chronic obstructive pulmonary disease (COPD). DBP has an indirect role in macrophage activation; thus it was hypothesised that DBP is present in the airway and contributes to lung disease by this mechanism.
\end{abstract}

Methods 471 PiZZ subjects with $\alpha 1$-antitrypsin deficiency (AATD) were genotyped for tag single nucleotide polymorphisms (SNPs) covering the DBP gene $(G C)$, together with known functional variants, prior to seeking association with COPD phenotypes. 140 subjects with usual COPD and 480 controls were available for replication. Vitamin $D$ and DBP levels were measured by tandem mass spectrometry and ELISA, respectively, in serum and DBP in the sol phase of sputum in a subset of 60 patients. Concentrations were related to phenotype and to alveolar macrophage activation.

Results rs2070741 was associated with airway bacterial colonisation $(p=0.04)$ and bronchiectasis $(p=0.01)$, as was rs7041 $(p=0.03)$ which also influenced vitamin $D$ concentrations ( $p=0.01$ ). The GC2 variant predisposed to bronchiectasis in AATD $(p=0.04)$ and protected against COPD $(p=0.05)$; the latter association was replicated in usual COPD versus controls $(p=0.04)$. Circulating DBP related inversely to forced expiratory volume in $1 \mathrm{~s}\left(\mathrm{FEV}_{1}\right)(\mathrm{p}=0.02)$, in direct contrast to vitamin $\mathrm{D}$, where deficiency related to low $\mathrm{FEV}_{1}(\mathrm{p}=0.04)$. Sol DBP related directly to alveolar macrophage activation ( $p=0.004)$.

Conclusions The genetic association of DBP with COPD may be mediated by effects on macrophage activation, since DBP relates to $\mathrm{FEV}_{1}$, and affects macrophage activation. Vitamin $\mathrm{D}$ effects may be independent of this, relating more strongly to innate immunity.

\section{INTRODUCTION}

Vitamin D insufficiency may be relevant to many diseases because of the role of vitamin $\mathrm{D}$ in modulating the innate and adaptive arms of the immune system. ${ }^{1}$ However, while mechanisms for vitamin $\mathrm{D}$ metabolism and signalling within immune function are well documented, relatively little is known about the impact of vitamin D-binding protein (DBP), a transport protein which is likely to be a pivotal factor in optimising the immune response to vitamin $\mathrm{D}^{2}$ In chronic obstructive pulmonary disease (COPD), low serum levels of 25 -hydroxyvitamin $\mathrm{D}_{3}\left(25 \mathrm{OHD}_{3}\right)$ have been reported ${ }^{3}$ and there is evidence implicating polymorphisms in the DBP gene $(G C)$ with COPD risk (reviewed elsewhere) ${ }^{4}$ and vitamin D deficiency. ${ }^{5}$
DBP is a glycosylated $\alpha$-globulin divided into two large domains (I and II) and a shorter domain at the C-terminus (domain III). ${ }^{6}$ It is expressed in many tissues, $^{7}$ and by neutrophils, ${ }^{8}$ contributes to macrophage activation, ${ }^{9}$ augments monocyte and neutrophil chemotaxis to C5-derived peptides and acts as an actin scavenger protein, as discussed in our recent review. ${ }^{4}$ In simplified terms, domain I binds vitamin $\mathrm{D}$ while domain III binds actin, functions which are independent of each other. In order to influence macrophage activation, DBP must be converted to macrophage-activating factor (DBP-MAF), by the action of enzymes released from lymphocytes. ${ }^{9}$ Increases in lymphocyte numbers in the lung are recognised in $\mathrm{COPD},{ }^{10}$ suggesting that this process will occur in the lung of subjects with COPD.

The DBP gene $(G C)$ is highly polymorphic with three common variants (GC1F, GC1S and GC2) and more than 120 rarer variants. ${ }^{11}$ Single nucleotide polymorphisms (SNPs; rs4588 and rs7041) in exon 11 result in the common isoforms, termed GC1 and GC2; GC1 is then subdivided into GC1F and GC1S. Their protein products differ as shown in table 1 , and appear to have functional consequences. rs7041 has been strongly linked to vitamin $\mathrm{D}$ levels in a recent genome-wide association study, ${ }^{5}$ and the GC2 protein is less able to be converted to DBPMAF than GC1 variants. ${ }^{12}$ Either, or both, of these functions might be relevant in COPD.

We hypothesised that DBP polymorphisms would associate with pulmonary function and infection-related phenotypes, based on known protein functions and prior work in usual COPD. We then sought to characterise the reasons for such genetic association by studying macrophage activation and vitamin $\mathrm{D}$ levels.

\section{METHODS \\ Patient populations}

Subjects with $\alpha 1$-antitrypsin deficiency (AATD)

A total of 471 unrelated Caucasian subjects from the UK national registry for AATD were studied. Ethical approval was given by the local ethics committee. All patients underwent full clinical assessment as described previously. ${ }^{13}$ The presence of bronchiectasis in four or more bronchopulmonary segments was taken to be a predominant bronchiectasis phenotype, as described previously, ${ }^{14}$ and bacterial colonisation of the airway by the presence of $>1 \times 10^{5} \mathrm{cfu} / \mathrm{ml}$ of potential pathogens present in sputum in the stable state. Exacerbation frequency was determined using self-reported symptoms according to the Anthonisen criteria ${ }^{15}$ and selfreported use of primary and secondary care services. 
Table 1 Haplotypes of rs7041 and rs4588 result in structurally different proteins

\begin{tabular}{lll}
\hline Variant & $\begin{array}{l}\text { Alleles at rs7041 } \\
\text { and rs4588 }\end{array}$ & $\begin{array}{l}\text { Protein composition at } \\
\text { positions 416 and 420 }\end{array}$ \\
\hline GC1F & $\mathrm{T} / \mathrm{C}$ & Asp/Thr \\
GC1S & $\mathrm{G} / \mathrm{C}$ & $\mathrm{Glu} / \mathrm{Thr}$ \\
GC2 & $\mathrm{T} / \mathrm{A}$ & Asp/Lys \\
\hline
\end{tabular}

The table shows the alleles present at rs7041 and rs4588, respectively (forward strand) and the resulting protein composition at positions 416 and 420 , respectively. In this manuscript, where the protein is discussed the nomenclature GC2, GC1S or GC1F is used in plain text, and when it refers to the underlying genetic code (haplotype) italics are used.

Of this cohort, 60 patients had stable state contemporaneous serum and sputum samples available and were selected for further study of DBP and vitamin D; specifically measurement of circulating $25 \mathrm{OHD}_{3}$ and $\mathrm{DBP}$, sputum $\mathrm{DBP}$ and alveolar macrophage (AM) function in the presence of DBP. Since large volumes of sputum are required to carry out all such assessments, only 22 patients had all measurements made.

\section{Replication cohort: usual COPD and controls}

The West Midlands COPD Collection has been set up to study COPD phenotypes and progression, with a focus on use of biological samples and data in exploring pathogenesis. Inclusion is based on spirometrically defined COPD, as described previously, ${ }^{16}$ although full phenotyping in the form of full lung function and CT scans is offered. Studies have been approved by the local ethics committee. At the time of data collection there were 140 Caucasian subjects registered, chosen to ensure racial matching with the AATD group. An anonymous, healthy, Caucasian, geographically matched control group $(n=480)$ were available for comparison. These individuals were not phenotyped, so no further details are known.

\section{Genotyping}

SNPs to tag GC were chosen using HapMap phase $\operatorname{III}^{17}$ to capture all SNPs with minor allele frequency $>0.05$ and $r^{2} \geq 0.8$. In addition the two SNPs that code for GC2, GC1F and GC1S (rs7041 and rs4588) were selected for study. DNA extraction, quantification and genotyping were carried out as described previously. ${ }^{18}$ All SNPs were genotyped in the subjects with AATD, with replication sought for associated variants in the case-control population.

\section{DBP and vitamin D measurement}

DBP was measured by ELISA (Immunodiagnostik, Bensheim, Germany) in serum and sputum. The assay was validated in sputum as described in the Supplementary material. Total $25 \mathrm{OHD}_{3}$ was measured by tandem mass spectrometry.

\section{Assessment of AM activity}

This was carried out using an efferocytosis assay, which assesses macrophage activation by means of the proportion of macrophages that engulf apoptotic neutrophils. This assay was chosen as a better model of neutrophilic inflammation in the COPD lung than a phagocytosis assay centred on bacteria or beads. AMs and neutrophils were obtained from individuals without COPD or AATD who were undergoing lung resection. Neutrophils were isolated by Percoll density centrifugation, ${ }^{19}$ incubated at $37^{\circ} \mathrm{C}$ for $20 \mathrm{~h}$, the trypan blue exclusion test and cell count were performed, and adequate signs of apoptosis were ascertained by cytospin and microscopy. Neutrophils were marked with cell tracker green and diluted to a concentration of $2 \times 10^{6} / \mathrm{ml}$ prior to use. The resected sample was flushed with saline and the wash centrifuged at $500 \mathrm{~g}$ for $5 \mathrm{~min}$ to obtain a cell pellet of AMs, which was underlayered with $12 \mathrm{ml}$ of lymphoprep. After further centrifugation at $800 \mathrm{~g}$ for $30 \mathrm{~min}$, the interphase was aspirated, the trypan blue exclusion test was performed and cells were counted. Samples were then washed with phosphatebuffered saline (PBS), diluted to $0.5 \times 10^{6}$ cells $/ \mathrm{ml}$ with $\mathrm{RPMI} / 10 \%$ fetal bovine serum and incubated at $37^{\circ} \mathrm{C}$ for $2 \mathrm{~h}$ prior to use.

For the final phase of the assay 400000 neutrophils were added to 100000 AMs in 48-well plates with either saline or the sol phase of sputum and incubated for $90 \mathrm{~min}$ at $37^{\circ} \mathrm{C}$. The supernatant was removed and the plate washed twice with cold PBS. A $100 \mu \mathrm{l}$ aliquot of cold serum-free RPMI was then added to the plate, which was promptly photographed with a fluorescence microscope. The total number of macrophages and the number that had ingested fluorescently marked neutrophils per field were counted and an efferocytosis index calculated (cells ingested/total cells). Each plate contained five saline controls, and five cell counts were performed per patient $(n=22)$. This was then repeated using pure DBP at the mean sol level, with an anti-DBP antibody (Sigma, Gillingham, UK).

\section{Statistical analysis}

Analyses were carried out in SPSS (version 16.0). In the genetic analyses, logistic and linear regression models were used, assuming additive effects, adjusting for age, gender and packyears smoked, with either COPD, bronchiectasis or exacerbation frequency as the dependent variable. In each case, adjustments were made based on known influences on each phenotype in this AATD cohort, as published previously. ${ }^{18}$ Data normality was assessed using the Kolmogorov-Smirnov test. Vitamin D and DBP were correlated with each other and with forced expiratory volume in $1 \mathrm{~s}\left(\mathrm{FEV}_{1}\right)$ using the Spearman rank correlation, and differences in their levels between alleles and genotypes tested using the Mann-Whitney and Kruskal-Wallis tests, respectively. The Kruskal-Wallis test was also used to compare $\mathrm{FEV}_{1}$ between vitamin $\mathrm{D}$ sufficiency groups. For results pertaining to cell counts, analysis of variance (ANOVA) was used. A significance threshold of $p<0.05$ was used throughout.

\section{RESULTS}

\section{Subject characteristics}

Characteristics of the subjects with AATD and COPD are shown in table 2. In the AATD group, 440/471 patients had undergone a CT scan and 75 stable state sputum samples were obtained. In the COPD group, 43/140 had undergone a CT scan, and though many reported sputum production none brought samples to their clinical assessment. The subset of patients with AATD in whom vitamin $\mathrm{D}$ and DBP measurements were made did not differ significantly in any demographic or clinical feature from the whole group (all $\mathrm{p}>0.1$ ).

\section{Genetic association analyses \\ Single SNPs}

In the AATD group, 12 SNPs were genotyped; 10 tag SNPs (capturing 28 SNPs) and two known to contribute to GC2, GC1F and GC1S. Their locations are shown in figure 1. Of these, $13 / 14$ were in Hardy-Weinberg equilibrium (the single SNP (rs3755967; $\mathrm{p}=0.04$ ) that was not was excluded from further analyses). The genotyping success rate overall was 95\%. Full genotype frequency data are shown in the Supplementary material. 
Table 2 Characteristics of the subjects with AATD and COPD

\begin{tabular}{|c|c|c|c|c|c|}
\hline Characteristic & $\begin{array}{l}\text { AATD } \\
(n=471)\end{array}$ & $\begin{array}{l}\text { AATD with COPD } \\
(\mathrm{n}=364)\end{array}$ & $\begin{array}{l}\text { AATD without COPD } \\
(\mathrm{n}=107)\end{array}$ & $\begin{array}{l}\text { AATD subset } \\
(n=60)\end{array}$ & $\begin{array}{l}\text { COPD } \\
(n=140)\end{array}$ \\
\hline Age & $50.33(10.35)$ & $51.75(9.33)$ & $43.46(36.37-56.36)$ & $49.71(9.88)$ & $68.82(0.93)$ \\
\hline Male gender & $281(59.70)$ & $233(64.00)$ & $57(53.27)$ & 37 (61.67) & $79(56.61)$ \\
\hline $\mathrm{FEV}_{1} \%$ predicted & $45.30(28.45-72.33)$ & $36.70(25.86-52.74)$ & $104.91(18.63)$ & $35.66(22.38-56.41)$ & $43.43(15.84)$ \\
\hline $\mathrm{FEV}_{1} / \mathrm{FVC}$ & $39.00(29.00-56.00)$ & $34.00(27.00-44.75)$ & $80.00(71.00-85.50)$ & $34.00(26.75-48.25)$ & $36.00(28.72-51.00)$ \\
\hline COPD & $364(78.62)$ & $364(100)$ & 0 & 49 (81.67) & $140(100)$ \\
\hline Exacerbations per year & $1.00(0-2)$ & $1.00(0-2)$ & $1.00(0-2)$ & $1.00(1-2)$ & $2.00(1-4)$ \\
\hline
\end{tabular}

The table shows the mean (SD) or median (IQR) for all quantitative outcomes, and number (\%) for the qualitative outcomes, these being gender, COPD, emphysema, bronchiectasis and airway bacterial colonisation. Mean (SD) is shown where data are normally distributed, and median (IOR) where they are non-normal.

*Percentages shown as a proportion of scans and sputum obtained, respectively.

AATD, $\alpha 1$-antitrypsin deficiency; COPD, chronic obstructive pulmonary disease; FEV 1 , forced expiratory volume in $1 \mathrm{~s}$; FVC, forced vital capacity.

Of the tag SNPs studied, one (rs2070741) showed allele and genotype association with bronchiectasis, the $\mathrm{C}$ allele conferring an OR of 1.80 (95\% CI 1.02 to 3.19); a second tag SNP showed an association only in the genotypic analysis (table 3). Stable state airway bacterial colonisation was also more likely in C allele carriers of rs2070741, even after adjustment for the presence of bronchiectasis (OR 3.84, 95\% CI 1.78 to 6.92, $\mathrm{p}=0.04$ ). Of the two SNPs that contribute to functional variants (rs4588 and rs7041) both tended to associate with bronchiectasis but only rs7041 reached statistical significance (table 3). Previous studies have suggested a role for rs7041 in COPD susceptibility; in this study it was not associated with $\mathrm{FEV}_{1}(\mathrm{p}=0.788)$ unless an interaction was specified between genotype and vitamin level $(\mathrm{p}=0.012)$. No associations with exacerbation frequency or COPD were seen.

\section{Haplotype analyses}

Since the functional difference in DBP only occurs with a combination of alleles at rs4588 and rs7041, we examined haplotypes that code for the protein variants GC2, GC1F and GC1S (table 1) for association with the primary outcomes. The GC2 variant associated with an increased risk of bronchiectasis (OR $1.51,95 \%$ CI 1.02 to $2.22, \mathrm{p}=0.034$ ), while no associations were seen for GC1F and GC1S. Conversely there was a decreased risk of COPD with the GC2 variant (OR 0.79, 95\% CI 0.65 to $0.99, p=0.048)$. Haplotype frequencies are shown in table 4 .

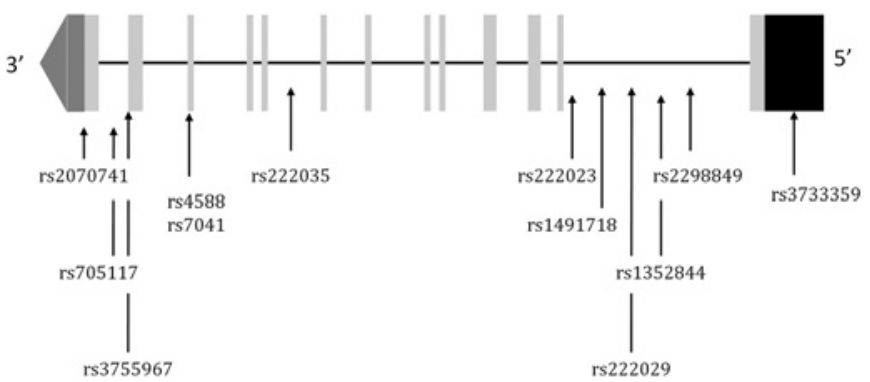

Figure 1 The structure of the vitamin D-binding protein (DBP) gene $(G C)$. GC consists of 13 exons, shown here as light grey vertical lines, with introns represented by a horizontal black line. The promoter is shown in black and other regulatory regions in dark grey. The location of the tag single nucleotide polymorphisms (SNPs) used and the nonsynonymous SNPs contributing to functional variants (rs4588 and rs7041) are shown.

\section{Replication data set}

The replication case:control group was genotyped for the three associated SNPs and rs4588 because of its contribution to GC2. Since few patients had undergone CT scans, we only tested for COPD as an outcome. The GC2 variant was protective (OR $0.73,95 \%$ CI 0.53 to $0.99, \mathrm{p}=0.042$ ).

\section{Vitamin D associations}

Vitamin D deficiency occurred in $45 \%$ of subjects and associated with lower $\mathrm{FEV}_{1}$ after adjustment for season of collection (figure 2 and Supplementary material). The $25 \mathrm{OHD}_{3}$ levels were lower in rs7041 T allele carriers $(p=0.010$, figure $3 \mathrm{~A})$, but no difference was seen with rs4588 $(p=0.411)$. There were few individuals homozygous for GC1S and none for GC1F, thus no valid interprotein comparisons of $25 \mathrm{OHD}_{3}$ level could be made. rs2070741 C allele carriers tended to have lower $25 \mathrm{OHD}_{3}$ $(p=0.052$; figure $3 \mathrm{~A})$ while there was no difference with rs2298849 ( $p=0.247)$.

\section{DBP associations}

\section{Serum DBP}

Serum DBP related inversely to $\mathrm{FEV}_{1}$ (figure $4 \mathrm{~A}$ ). Although this relationship was the opposite of that seen for vitamin $\mathrm{D}$ with $\mathrm{FEV}_{1}$, no relationship between the two was seen (figure 4B). Serum DBP level did not differ with GC genotype in SNP or haplotype analyses (all $\mathrm{p}>0.6$ ).

\section{Sol phase DBP}

DBP was present in sputum (mean (SE) $0.31(0.02) \mathrm{mg} / \mathrm{dl}$ ), but did not correlate with serum DBP $(\sigma=-0.03, p=0.91)$. Sol phase DBP was higher with the Tallele of $r 57041$ ( $p=0.037$, figure 3B) and did not vary with other associated SNPs or haplotypes (all $\mathrm{p}>0.8)$.

Table 3 Single nucleotide polymorphism (SNP) associations with bronchiectasis in $\alpha 1$-antitrypsin deficiency

\begin{tabular}{lllll}
\hline & $\begin{array}{l}\text { Genotype } \\
\text { model (p) }\end{array}$ & $\begin{array}{l}\text { Allele } \\
\text { model (p) }\end{array}$ & Allele $\mathbf{~ O R}$ & $\mathbf{9 5 \%}$ Cl \\
\hline rs2070741 $[\mathrm{A} / \mathrm{C}]$ & 0.031 & 0.046 & 1.80 & 1.02 to 3.19 \\
rs2298849 $[\mathrm{C} / \mathrm{T}]$ & 0.013 & 0.221 & - & - \\
rs4588 [C/A] & 0.280 & 0.067 & - & - \\
rs7041 [G/T] & 0.526 & 0.027 & 0.52 & 0.29 to 0.93
\end{tabular}

The table shows the $\mathrm{p}$ values and $\mathrm{OR}$ and $95 \% \mathrm{Cl}$ of bronchiectasis for each SNP; in each case the OR was computed for the minor allele. All results with $p<0.1$ are shown. No OR has been computed for rs 2298849 and rs 4588 since the allele analyses were non-significant. 
Table 4 GC haplotypes observed in AATD, COPD and controls

\begin{tabular}{llll}
\hline & AATD & COPD & Control \\
\hline GC2-GC2 & $41(10.3)$ & $10(8.9)$ & $56(12.9)$ \\
GC1S-GC1S & $110(27.6)$ & $39(25.9)$ & $145(33.5)$ \\
GC1F-GC1F & $1(0.2)$ & 0 & 0 \\
GC2-GC1S & $142(35.6)$ & $41(36.6)$ & $150(34.6)$ \\
GC2-GC1F & $5(1.3)$ & $1(0.8)$ & 0 \\
GC1S-GC1F & $4(1.0)$ & $2(1.6)$ & 0 \\
\hline
\end{tabular}

Haplotype frequencies are shown as the raw value with the percentage in parentheses. Note that protein structure Glu/Lys would result from the haplotype rs7041G and rs $4588 \mathrm{~A}$; only the recognised haplotypes are listed here, thus percentages do not total $100 \%$. Where the genotype at either rs7041 or rs4588 was undetermined no haplotype has been assigned, and the individual was not included in the percentage analyses.

AATD, $\alpha 1$-antitrypsin deficiency; COPD, chronic obstructive pulmonary disease.

\section{Macrophage activation}

Sputum from patients with AATD increased macrophage activation as measured by efferocytosis of apoptotic neutrophils. The degree of activation related directly to the amount of DBP present (figure $5 \mathrm{~A}$ ). In control experiments using physiologically relevant concentrations of DBP alone, the same relationship was seen in multiple repeats of the experiment, suggesting that DBP is the component of sol phase responsible for the relationship (figure $5 \mathrm{~B}$ ). Activation could be reduced by $30 \%$ after addition of an anti-DBP antibody, but not completely abrogated (see also the Supplementary material).

No differences in activation according to GC genotype were seen (all $\mathrm{p}>0.3$ ). Protein variant analyses could not be carried out in a meaningful manner due to lack of homozygous subjects in this subset.

\section{DISCUSSION}

This study demonstrates lower $\mathrm{FEV}_{1}$ in the presence of high DBP, and increased macrophage activation with high airway levels of DBP. The GC2 variant, which is less able to activate macrophages, also exhibited genetic association with a decreased risk of COPD. Together these results suggest that increased macrophage activation due to DBP may be deleterious to lung function and relevant to the pathogenesis of COPD. In addition there was an increased risk of bronchiectasis in patients with AATD with GC2, perhaps because of a reduced ability to defend the airway against pathogens. Differences in vitamin $\mathrm{D}$ level are also associated with underlying GC genotypes, and could be
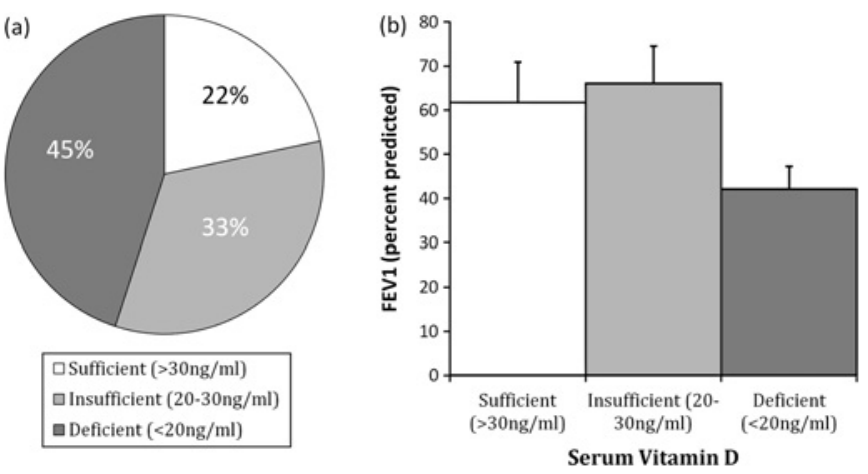

Figure 2 Vitamin $D$ deficiency is common in $\alpha 1$-antitrypsin deficiency (AATD) and relates to lung function. The incidence of vitamin D deficiency is shown in the pie chart $(A)$, and related to forced expiratory volume in $1 \mathrm{~s}\left(\mathrm{FEV}_{1}\right)(\mathrm{p}=0.04)(\mathrm{B})$. The column chart shows the mean $\mathrm{FEV}_{1}$ (error bars represent $\mathrm{SE}$ ) and serum vitamin D level, as categorised after adjustment for season of collection.
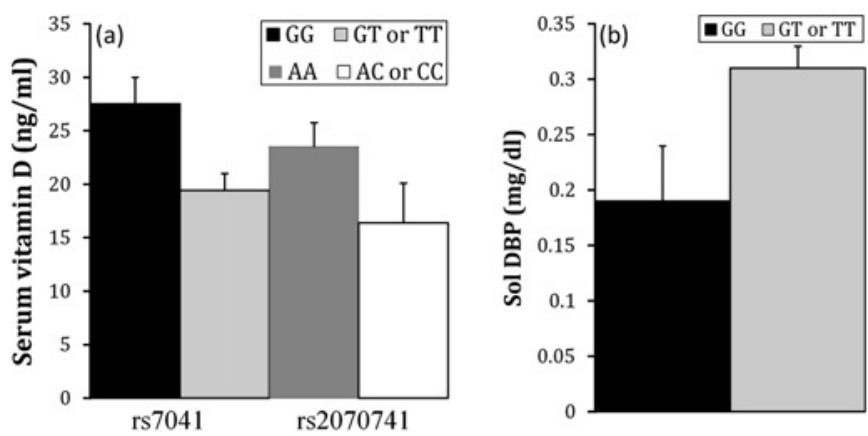

Figure 3 Relationship of GC single nucleotide polymorphism (SNP) genotypes to vitamin $D$ and vitamin $D$-binding protein (DBP) in $\alpha 1$-antitrypsin deficiency (AATD). Graphs show the mean (SE) for the genotypes listed in the legends. (A) Vitamin D is lower in carriers of the T allele of $r$ 7041 $(p=0.01)$ and tends to be lower in carriers of the $C$ allele of $r$ 2070701 $(p=0.052)$. (B) DBP is higher in the airway in carriers of the T allele of rs7041 ( $p=0.037)$.

contributing to pathogenesis, although such mechanisms were not the focus of this study. These concepts are illustrated in figure 6.

\section{What are the possible roles for DBP in COPD pathogenesis?}

Macrophages accumulate in the COPD lung, and could be harmful if activated because of release of neutrophil chemotractants. $^{20}$ Augmentation of proteolytic damage might also occur in smokers because smoke increases matrix metalloprotease transcription by alveolar macrophages. ${ }^{21}$ This potential harm has to be balanced against the benefit of scavenging damaged tissue and infectious organisms, processes which may paradoxically be decreased in COPD. ${ }^{22}$ The genetic association seen here suggests that macrophage activation secondary to DBP has adverse consequences in the lung, as illustrated in figure 6.

A limitation of this study is that we were unable to find a neutralising antibody that completely inhibited the activity of DBP. This may be because of high or unstable DBP levels in the experiments. Neutrophil elastase is capable of cleaving DBP from cell surface binding sites ${ }^{23}$; use of apoptotic neutrophils here should have limited this, but we cannot exclude an effect. It is also unknown how quickly DBP converts to DBP-MAF in these experimental conditions - it may be necessary to design an antibody to DBF-MAF to ascertain if this improves the effect size seen. In addition, few patients were studied using efferocytosis, and none in this group was homozygous for GC2, thus
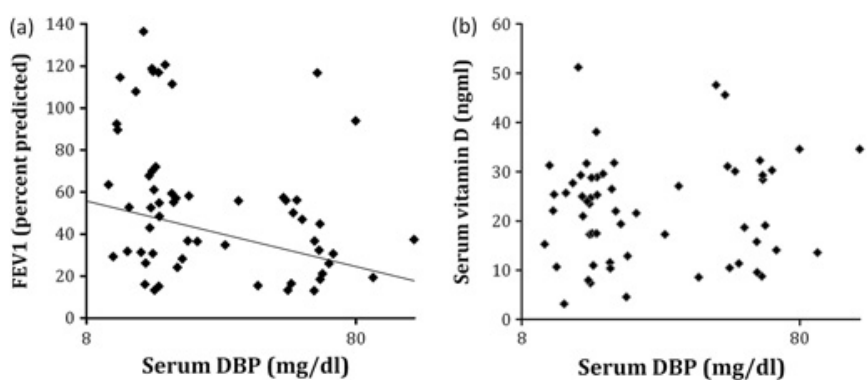

Figure 4 Serum vitamin D-binding protein (DBP) correlates inversely with forced expiratory volume in $1 \mathrm{~s}\left(\mathrm{FEV}_{1}\right)$ but is not related to vitamin $D$ in $\alpha 1$-antitrypsin deficiency (AATD). (A) $\mathrm{FEV}_{1}$ was lower in the presence of high circulating DBP; Spearmans rank correlation $(\sigma)=-0.32, p=0.02$. (B) DBP did not relate to vitamin $D, \sigma=0.09, p=0.52$. 

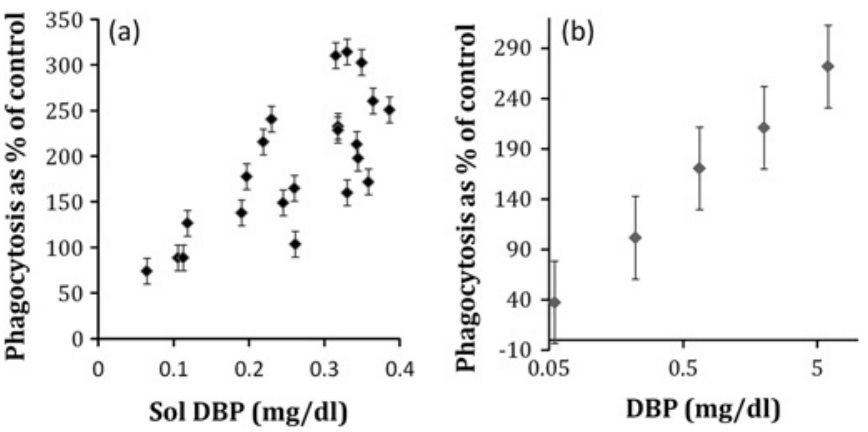

Figure 5 Sputum vitamin D-binding protein (DBP) correlates directly with macrophage activation. The scatterplot shows the mean (SE) of the phagocytosis index, expressed as a percentage of the negative control, this being a measure of macrophage activity. (A) The degree of phagocytosis related strongly to DBP in subjects' sputum $(p=0.004$ by analysis of variance). The mean sol DBP for the group was $0.31 \mathrm{mg} / \mathrm{dl}$, at which the macrophage activation was elevated to $150 \%$ of the control, indicating increased activation in the majority of subjects. (B) When DBP standards were used, the same relationship was seen $(p<0.001)$.

the link between genetics and altered macrophage activation could not be shown directly, although this has been established by others. ${ }^{12}$ The association with bronchiectasis could not be replicated due to low take up of CT in usual COPD, but would be consistent with the effect of DBP on macrophages.

The other functions of DBP relevant to COPD are its influence on neutrophil chemotaxis and actin binding. DBP is not directly chemotactic to neutrophils, but enhances the chemotactic effect of complement-derived peptides (C5a). ${ }^{24}$ The level of DBP in bronchoalveolar lavage fluid (BALF) from subjects with COPD relates directly to chemotaxis, which can be reduced by an antiDBP antibody, confirming that this is relevant to pathogenesis. ${ }^{25}$ Concentrations of DBP in BALF were lower than we saw in sputum, which may account for the differences in antibody effect compared with the current study. Genetic variation has

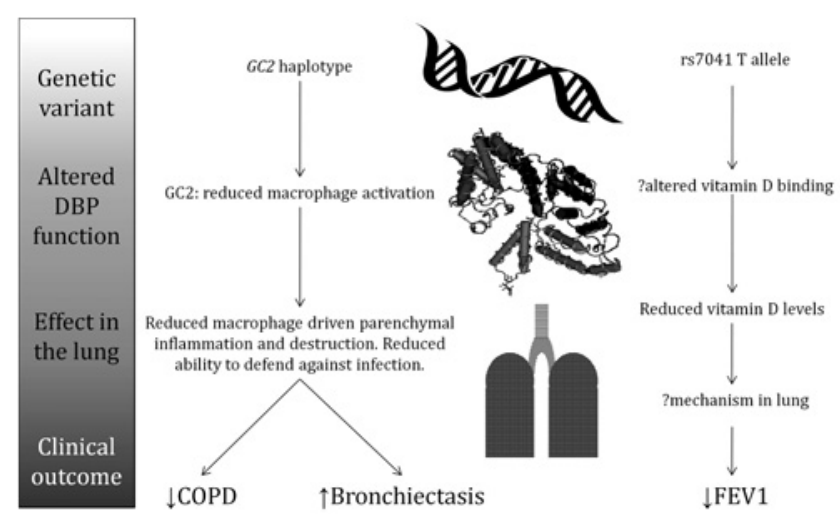

Figure 6 Proposed consequences of variation in vitamin D-binding protein (DBP) in the lung. The GC2 haplotype codes for the GC2 protein variant, which is less able to be converted to DBP-macrophageactivating factor (MAF), resulting in decreased macrophage activation in the lung, which could lead to brochiectasis, or protect against chronic obstructive pulmonary disease (COPD). rs7041 results in a single amino acid change in DBP, which could conceivably influence vitamin $D$ binding, and explain the observation of low vitamin $D$ levels in carriers of this allele. The subsequent mechanism in the lung is as yet unknown, but in the current study the interaction between genotype and vitamin level was associated with low forced expiratory volume in $1 \mathrm{~s}\left(\mathrm{FEV}_{1}\right)$. not been shown to influence neutrophil chemotaxis or actin binding, hence these functions were not chosen for study here.

\section{How could vitamin D contribute to COPD pathogenesis?}

Vitamin D deficiency was associated with reduced lung function, and was predicted by the presence of the Tallele of rs7041, confirming the recent findings of Janssens et al. ${ }^{3}$ rs7041 is not known to influence macrophage function unless as part of a haplotype with rs4588, suggesting that an alternative mechanism is at work. The polymorphism results in an amino acid change in domain 1 of DBP, such that altered binding of vitamin $\mathrm{D}$ to DBP could occur. This may influence availability of vitamin $\mathrm{D}$ to cells by altering endocytosis of $25 \mathrm{OHD}_{3}$ bound to DBP, which occurs via interaction with cell surface proteins ${ }^{26}$ in many cell types, including macrophages. ${ }^{27}$ This interaction has been demonstrated in vitro, ${ }^{2}$ and has been suggested epidemiologically to confer risk of tuberculosis. ${ }^{28}$ In the current study we demonstrated that the interaction term between genotype and vitamin $\mathrm{D}$ level related to $\mathrm{FEV}_{1}$, providing additional support for this hypothesis. Further study of this as a biological mechanism, independent of macrophage activation, is now indicated. The remaining SNP associations (rs2070741 and rs2298849) must be viewed with caution since they have not been replicated. Neither SNP has any known function, although the trend seen here to lower vitamin $\mathrm{D}$ levels with the associated allele of rs2070741 suggests a similar mechanism to rs7041.

It is also interesting to speculate why high sol DBP occurred with a variant linked to vitamin $\mathrm{D}$ deficiency. This study cannot imply causality without further functional genetic work, but a logical explanation might be that vitamin D-deficient subjects are more prone to respiratory infection and hence inflammation. ${ }^{1}$ Inflammatory cytokines stimulate transcription of $\mathrm{DBP},{ }^{29}$ such that airway concentrations are then elevated. This interpretation is more consistent with the genetic association than a direct influence of rs7041 on DBP, since levels did not differ with genotype, and would not be expected to do so, as it is has no known influence on transcription. Compartment-specific genetic differences are possible, however, as we have recently shown with circulating and sol phase tumour necrosis factor $\alpha,{ }^{1830}$ and this will require further study.

\section{Limitations}

The study is limited by small numbers for the genetic association analyses, and lack of CT phenotyping data and stable state microbiology in the usual COPD group. The controls in the COPD analysis are also non-phenotyped, which may be perceived as a weakness, although it is a recognised methodology in large-scale genetic association work. ${ }^{31}$ Small cohorts may also be problematic when calculating allele frequencies, which may appear to fluctuate markedly if insufficient numbers of people are considered. The frequency of the minor allele of rs7041 was 0.28 in controls and 0.43 for rs 4588 . These compare with means of 0.42 for rs 7041 and 0.32 for rs4588 in Caucasian cohorts reported in $\mathrm{dbSNP}^{32}$; consistent with this, frequencies of minor allele homozygotes for rs7041 are lower in our cohorts than those observed by Jansenns et $a l^{\beta}$ in both COPD and control subjects. A knock on effect of the relatively low $\mathrm{T}$ allele frequency is a low frequency of the GC2 haplotype. Nevertheless, we are confident of the veracity of our results in this population because of our quality control procedures for genotyping, and because we replicate the association of the $T$ allele of rs7041 with vitamin D levels.

The sputum studies could only be carried out in a subset who produced sputum regularly in the stable state and the 
efferocytosis experiments only on the largest volume samples. Although this could produce bias towards chronic bronchitics and bronchiectatics, it is important to note that this subset did not differ significantly in their clinical parameters from the whole. In the COPD group, take up of specialist tests and sputum provision was low. We might speculate that this is due to greater difficulties in getting to hospital for tests due to age and co-morbidity.

\section{Conclusion}

$\mathrm{DBP}$ and vitamin $\mathrm{D}$ both play a role in the pathogenesis of COPD, and may do so via independent pathways. Detailed characterisation of all components of the vitamin $\mathrm{D}$ axis will be necessary to dissect these effects further and ascertain if supplementation of vitamin $\mathrm{D}$ will be beneficial.

Acknowledgements We would like to thank all members of the UK AATD registry, their funders (Talecris Biotherapeutics (RAS), the Wellcome trust (DRT), the West Midlands Chest Fund (AMW, PRN) and NIHR (CB)) as well as the West Midlands COPD Collection investigating teams for gathering of clinical data and biological samples. In addition we would like to thank the Midlands Lung Tissue Collaborative at Birmingham Heartlands Hospital for their assistance with lung tissue collection.

Funding Talecris Biotherapeutics (non-commercial grant) and the West Midlands Chest Fund funded the majority of this work. DRT is also funded by the Wellcome Trust.

\section{Competing interests None.}

Ethics approval This study was conducted with the approval of the South Birmingham LREC refs. 3359, 3359a and 07/H1207/231, and North West REC ref. 07/MRE08/42.

Contributors AMW conceived the study, performed AATD genotyping, DBP measurements and statistical analysis, and drafted the paper. CRB and DW performed the efferocytosis assays. PRN performed control and COPD genotyping. DRT and RAS supervised the work and reviewed the manuscript.

Provenance and peer review Not commissioned; externally peer reviewed.

\section{REFERENCES}

1. Adams JS, Hewison M. Unexpected actions of vitamin D: new perspectives on the regulation of innate and adaptive immunity. Nat Clin Pract Endocrinol Metab 2008:4:80-90.

2. Chun RF, Lauridsen AL, Suon L, et al. Vitamin D-binding protein directs monocyte responses to 25-hydroxy- and 1,25-dihydroxy vitamin D. J Clin Endocrinol Metab 2010;95:3368-76

3. Janssens W, Bouillon $R$, Claes $B$, et al. Vitamin D deficiency is highly prevalent in COPD and correlates with variants in the vitamin D-binding gene. Thorax 2010;65:215-20.

4. Chishimba L, Thickett DR, Stockley RA, et al. The vitamin D axis in the lung: a key role for vitamin D-binding protein. Thorax 2010;65:456-62.

5. Ahn J, Yu K, Stolzenberg-Solomon R, et al. Genome-wide association study of circulating vitamin D levels. Hum Mol Genet 2010:19:2739-45.

6. Swamy N, Head JF, Weitz D, et al. Biochemical and preliminary crystallographic characterization of the vitamin $D$ sterol- and actin-binding by human vitamin D-binding protein. Arch Biochem Biophys 2002:402:14-23.

7. McLeod JF, Cooke NE. The vitamin D-binding protein, alpha-fetoprotein, albumin multigene family: detection of transcripts in multiple tissues. J Biol Chem 1989:264:21760-9.
8. Kew RR, Sibug MA, Liuzzo JP, et al. Localization and quantitation of the vitamin D binding protein (Gc-globulin) in human neutrophils. Blood 1993;82:274-83.

9. Yamamoto N, Homma S. Vitamin D3 binding protein (group-specific component) is a precursor for the macrophage-activating signal factor from lysophosphatidylcholinetreated lymphocytes. Proc Natl Acad Sci USA 1991;88:8539-43.

10. Hogg JC, Chu F, Utokaparch $\mathrm{S}$, et al. The nature of small-airway obstruction in chronic obstructive pulmonary disease. N Engl J Med 2004;350:2645-53.

11. Cleve $\mathbf{H}$, Constans J. The mutants of the vitamin-D-binding protein: more than 120 variants of the GC/DBP system. Vox Sang 1988;54:215-25.

12. Viau M, Constans J, Debray $\mathrm{H}$, et al. Isolation and characterization of the 0-glycan chain of the human vitamin-D binding protein. Biochem Biophys Res Commun 1983:117:324-31.

13. Dowson LJ, Guest PJ, Hill SL, et al. High-resolution computed tomography scanning in alpha1-antitrypsin deficiency: relationship to lung function and health status. Eur Respir J 2001:17:1097-104.

14. Parr DG, Guest PG, Reynolds JH, et al. Prevalence and impact of bronchiectasis in alpha1-antitrypsin deficiency. Am J Respir Crit Care Med 2007:176:1215-21.

15. Anthonisen NR, Manfreda J, Warren CP, et al. Antibiotic therapy in exacerbations of chronic obstructive pulmonary disease. Ann Intern Med 1987:106:196-204.

16. Wood AM, de Pablo P, Ahmad A, et al. Smoke exposure as a determinant of autoantibody titre in AATD and COPD. Eur Respir J Published Online First: 4 June 2010. doi:10.1183/09031936.00033710.

17. The International HapMap Consortium. A haplotype map of the human genome Nature 2005:437:1299-320.

18. Wood AM, Simmonds MJ, Bayley DL, et al. The TNFalpha gene relates to clinical phenotype in alpha-1-antitrypsin deficiency. Respir Res 2008;9:52.

19. Jepsen LV, Skottun T. A rapid one step method for the isolation of human granulocytes from whole blood. Scand J Clin Lab Invest 1982;42:235-8.

20. Finney-Hayward TK, Bahra P, Li S, et al. Leukotriene B4 release by human lung macrophages via receptor not voltage operated $\mathrm{Ca} 2+$ channels. Eur Respir $\mathrm{J}$ 2009;35:1105-12.

21. Shaykhiev R, Krause S, Salit J, et al. Smoking dependent reprogramming of alveolar macrophage activation: implication for pathogenesis of chronic obstructive pulmonary disease. J Immunol 2009;183:2867-83.

22. Berenson CS, Wrona CT, Grove LJ, et al. Impaired alveolar macrophage response to Haemophilus antigens in chronic obstructive pulmonary disease. Am J Respir Crit Care Med 2006;174:31-40

23. DiMartino SJ, Shah $A B$, Trujillo $G$, et al. Elastase controls the binding of the vitamin D-binding protein (Gc-globulin) to neutrophils: a potential role in the regulation of C5a co-chemotactic activity. J Immunol 2001;166:2688-94.

24. Zhang J, Kew RR. Identification of a region in the vitamin D-binding protein that mediates its C5a chemotactic cofactor function. J Biol Chem 2004;279:53282-7.

25. Metcalf JP, Thompson AB, Gossman GL, et al. Gc globulin functions as a cochemotaxin in the lower respiratory tract. A potential mechanism for lung neutrophil recruitment in cigarette smokers. Am Rev Respir Dis 1991;143:844-9.

26. Nykjaer A, Dragun D, Walther D, et al. An endocytic pathway essential for renal uptake and activation of the steroid 25-(OH) vitamin D3. Cell 1999;96:507-15.

27. Chun RF, Adams JS. Back to the future: a new look at 'old' vitamin D. J Endocrinol 2008;198:261-9.

28. Martineau AR, Leandro AC, Anderson ST, et al. Association between Gc genotype and susceptibility to TB is dependent on vitamin D status. Eur Respir $J$ 2010;35:1106-12.

29. Guha C, Osawa M, Werner PA, et al. Regulation of human Gc (vitamin D-binding) protein levels: hormonal and cytokine control of gene expression in vitro. Hepatology 1995;21:1675-81.

30. Sapey E, Wood AM, Ahmad A, et al. TNF $\{$ alpha\} rs361525 polymorphism is associated with increased local production and downstream inflammation in COPD. Am J Respir Crit Care Med 2010;182:192-9.

31. WTCCC. Genome-wide association study of 14,000 cases of seven common diseases and 3,000 shared controls. Nature 2007;447:661-78.

32. Sherry ST, Ward MH, Kholodov M, et al. dbSNP: the NCBI database of genetic variation. Nucleic Acids Res 2001:29:308-11. 